\title{
ON A CONJECTURE BY M. OZAWA CONCERNING FACTORIZATION OF ENTIRE FUNCTIONS
}

\author{
W. H. J. FUCHS and G. D. SONG
}

0 . In a series of four papers [2, 3] M. Ozawa considered entire functions $F(z)$ possessing, for infinitely many $k$, a factorization

$$
F(z)=P_{k} \circ g_{k}(z)
$$

where $P_{k}$ is a polynomial of degree $k$ and $g_{k}$ is an entire function. He proved

Theorem A. If (1) holds for $k=2^{j}(j=1,2, \ldots)$ and for either $k=3$ or $k=5$, then either

$$
F(z)=a e^{H(z)}+b \quad(a, b \in C, H(z) \text { entire })
$$

or

$$
F(z)=a \cos \left((H(z))^{1 / 2}\right)+b .
$$

Indeed for a function of the form (2)

$$
F(z)=\left(a u^{n}+b\right) \circ e^{H(z) / n}
$$

shows that (1) holds for $k=1,2,3, \ldots$. And if the polynomial $T_{n}$ is defined by

$$
T_{n}(\cos \theta)=\cos n \theta,
$$

then a function of the form (3) satisfies

$$
F(z)=a T_{n}\left(\cos \frac{H(z)^{1 / 2}}{n}\right)+b \quad(n=1,2, \ldots)
$$

and again (1) is true for $k=1,2, \ldots$.

Ozawa also proved

Theorem B. If (1) holds for $k=3^{j}, k=2$ and $k=4$, then $F(z)$ must be either of the form (2) or of the form (3).

These results led Ozawa to the

Conjecture. If (1) holds for $k=q \geqq 2$ and $k=n_{j} \geqq 2$, where $n_{j}$ divides $n_{j+1}$ and $\left(q, n_{j}\right)=1(j=1,2, \ldots)$, then the conclusion of Theorem A holds. 
We shall show that this conjecture is not generally correct by proving

Theorem 1. There are entire functions $F(z)$ which are not of the form (2) or (3) and which satisfy (1) for $k=n \geqq 2, k=q \geqq 2,(n, q)=1$, and for $k=(1+n q)^{l}$ $(l=1,2, \ldots)$.

However, if the sequence $n_{j}$ does not increase too rapidly, then the conjecture is correct.

We shall prove

Theorem 2. If the entire function $F(z)$ satisfies (1) for $k=n_{1}, k=q$ $\left(2 \leqq n_{1}<q,\left(n_{1}, q\right)=1\right)$ and for $k=n_{j}(j=2,3, \ldots)$ where $n_{j+1} \leqq n_{j} q, \quad\left(n_{j}, q\right)=1$, then $F(z)$ is either of the form (2) or of the form (3).

\section{Proof of Theorem 1. Let}

Let

$$
Q_{k}(z)=z+z^{n q+1} / c_{k} \quad\left(c_{k}>0\right) .
$$

$$
h_{v, m}(z)=Q_{v+1} \circ \ldots \circ Q_{m}(z) \quad(m>v \geqq 0) .
$$

We prove first that the constants $c_{k}$ can be chosen so that

$$
h_{v}(z)=\lim _{m \rightarrow \infty} h_{v m}(z)
$$

is an entire function for every $v \geqq 1$.

As the first step in the construction we choose

$$
c_{1}=1, Q_{1}(z)=z+z^{n q+1} .
$$

Suppose $Q_{1} \ldots Q_{k}$ have already been chosen. Put

$$
A_{k}=\max _{0 \leqq v \leqq k-1} \sup _{\substack{|z| \leq k \\\left|z^{\prime}\right| \leqq k+1}}\left|\frac{h_{v, k}\left(z^{\prime}\right)-h_{v, k}(z)}{z^{\prime}-z}\right|,
$$

with the obvious definition of the right hand side for $z=z^{\prime}$.

Let $c_{k+1}=\left[2^{k} k^{n q+1} \max \left(1, A_{k}\right)\right]$. Then, in $|z| \leqq k$,

$$
\left|Q_{k+1}(z)\right|<|z|+\frac{k^{n q+1}}{2^{k} k^{n q+1}}<|z|+1 .
$$

For $v \quad:-1,|z| \leqq k$, by the definition of $A_{k}$

$$
\left|h_{v, k+1}(z)-h_{v, k}(z)\right| \leqq A_{k}\left|Q_{k+1}(z)-z\right| \leqq \frac{k^{n q+1}}{2^{k} k^{n q+1}}=2^{-k} .
$$

Therefore

$$
\lim _{k \rightarrow \infty} h_{v, k}(z)=h_{v, v+1}(z)+\sum_{k=v+1}^{\infty}\left\{h_{v, k+1}(z)-h_{v, k}(z)\right\}
$$


is uniformly convergent in $|z|<m$ for every $m>0$, i.e.

$$
h_{v}(z)=\lim _{k \rightarrow \infty} h_{v, k}(z)
$$

is an entire function. Obviously

where

$$
h_{0}(z)=R_{v} \circ h_{v}(z) \quad(v \geqq 1)
$$

$$
R_{v}=Q_{1} \circ Q_{2} \ldots \circ Q_{v}
$$

is a polynomial of degree $(1+n q)^{v}$.

The functions $h_{v}(z)$ are not constants, because it follows from the definition that

$$
h_{v}(1) \geqq 1, h_{v}(0)=0 \quad(v=0,1,2 \ldots) .
$$

By induction on $v$ it is easily seen that

$$
R_{v}(u)=u k_{v}\left(u^{n q}\right)
$$

where $k_{v}$ is a polynomial of degree

$$
\operatorname{deg} k_{v}=\frac{1}{n q}\left((1+n q)^{v}-1\right) \text {. }
$$

Choose

$$
F(z)=\left(h_{0}(z)\right)^{n q}, g_{v}(z)=\left(h_{v}(z)\right)^{n q} \text {. }
$$

Then

$$
\begin{aligned}
F(z)=u^{n} \circ\left(h_{0}(z)\right)^{q} & =u^{q} \circ\left(h_{0}(z)\right)^{n}=\left(R_{v} \circ h_{v}(z)\right)^{n q}=\left(h_{v}(z) k_{v}\left(h_{v}(z)^{n q}\right)\right)^{n q} \\
& =u\left(k_{v}(u)\right)^{n q} \circ g_{v}(z)=P_{v} \circ g_{v}(z),
\end{aligned}
$$

where $P_{v}$ is a polynomial of degree

$$
1+n q \cdot \operatorname{deg} k_{v}=(1+n q)^{v} .
$$

The function $F(z)$ has all the required factorizations.

We must still show that $F(z)$ is not of the form (2) or (3). The equation

$$
q(u)=u\left(k_{1}(u)\right)^{n q}=u(1+u)^{n q}=\alpha
$$

has at least 2 distinct roots for every value of $\alpha$, since $q^{\prime}(u)$ is not a perfect power. The entire function $g_{1}(z)$ omits at most one value. Therefore $F(z)=u\left(k_{1}(u)\right)^{n q} \circ g_{1}(z)$ assumes every value and so $F(z)$ is not of the form (2).

Suppose

$$
F(z)=\left(h_{0}(z)\right)^{n q}=a \cos \sqrt{H(z)}+b .
$$

Choose $\gamma$ so that $a \cos \gamma+b=0$. If $z_{1}$ is a root of multiplicity $l$ of

$$
H(z)=(\gamma+2 k \pi)^{2} \neq 0 \quad(k \in Z),
$$

then the power series of $\sqrt{H(z)}$ in powers of $z-z_{1}$ is

$$
\sqrt{H(z)}= \pm(\gamma+2 k \pi)+\alpha\left(z-z_{1}\right)^{l}+\ldots \quad(\alpha \neq 0) .
$$


By Taylor's theorem

$$
a \cos \sqrt{H(z)}+b=a \cos \gamma+b+\beta\left(z-z_{1}\right)^{l}+\ldots=\beta\left(z-z_{1}\right)^{l}+\ldots
$$

where $\beta \neq 0$, if $\gamma \neq 0(\bmod \pi)$. If $\gamma \equiv 0(\bmod \pi)$, then

$$
a \cos \sqrt{H(z)}+b=\beta\left(z-z_{1}\right)^{2 l}+\ldots \quad \beta \neq 0 .
$$

By (5) every zero of $a \cos \sqrt{H(z)}+b$ must have a multiplicity divisible by $n q \geqq 6$. Hence every root of (6) has multiplicity $l \geqq 3$, the value $(\gamma+2 k \pi)^{2}$ of $H(z)$ is completely ramified. But a well known theorem of Nevanlinna theory [1, p. 44] asserts that an entire function has at most 2 completely ramified values. This contradicts (5) and the proof of Theorem 1 is completed.

2. Some preliminary results. Our proof, like Ozawa's work, is based on

Picard's theorem. (cf. [4].) Let $R(u, v)$ be an irreducible polynomial in $C[u, v]$. If there are non-constant entire functions $f(z), g(z)$ such that

$$
R(f(z), g(z))=0 \quad(\forall z \in C),
$$

then the Riemann surface defined by

$$
R(u, v)=0
$$

is of genus zero.

(The Theorem is usually stated in the form: If (7) holds for meromorphic functions $f, g$, then $R(u, v)=0$ defines a Riemann surface of genus $\leqq 1$. But Riemann surfaces of genus one can only be uniformized by elliptic functions, not by entire functions.)

A Riemann surface $X$ of genus 0 is conformally equivalent to the Riemann sphere, i.e., its points can be put into $1-1$ correspondence with a parameter $s$ ranging over the Riemann sphere so that any holomorphic function on $X$ can be written as a holomorphic function of $s$ defined on the Riemann sphere, that is to say a rational function of $s$. In particular the points of the surface (8) are in 1-1 correspondence with the points $s$ of the Riemann sphere by

$$
u=U(s), \quad v=V(s) \quad(U, V \text { rational }) .
$$

Note that the parameter $s$ can be replaced by any fractional linear transform $T$ of $s$, if $U$ and $V$ are changed into $U \circ T^{-1}, V \circ T^{-1}$.

Lemma 1. If $f(z)$ and $g(z)$ are non-constant entire functions and if $P_{m}$ and $P_{n}$ are polynomials of relatively prime degrees $m, n$ respectively, then the identity

$$
P_{m} \circ f(z)=P_{n} \circ g(z) \quad(\forall z \in C)
$$

implies the existence of an entire function $s(z)$ and of polynomials $U$ (of degree $n$ ) and $V$ (of degree $m$ ) such that

$$
f(z)=U(s(z)), \quad g(z)=V(s(z)) .
$$


Proof of Lemma 1. Factorize

$$
P_{m}(u)-P_{n}(v)
$$

into irreducible factors in $C[u, v]$. If (10) holds, then one of these irreducible factors, $R(u, v)$, say, will satisfy

$$
R(f(z), g(z))=0 .
$$

By Picard's theorem this means that there is a conformal map $s=\psi(p)$ of the points $p$ of the Riemann surface $X$ of

$$
R(u, v)=0
$$

onto the points $s$ of the Riemann sphere. Without loss of generality we may assume that $s=\infty$ corresponds to a point with $u=\infty$.

Except at a finite number of branch points of $R$ we may use $u$ as local uniformizing parameter, so that $s$ is a holomorphic function $\sigma(u)$ of $u$ near all points of $R$ except the branch points. Therefore the map

$$
z \mapsto(f(z), g(z))=p \mapsto s=\psi(p)=\sigma \circ f(z)=s(z)
$$

is holomorphic near all $z$ except perhaps those for which $(f(z), g(z))=\left(u_{1}, v_{1}\right)=p_{1}$ is a branch point of $X$. These values of $z$ form a discrete set $E$. If $z \rightarrow z_{1} \in E, s(z) \rightarrow s\left(p_{1}\right)$. Therefore $z_{1}$ is a removable singularity of $s(z), s(z)$ is entire.

By (9), on $X$

and so

$$
u=U(s), \quad v=V(s)
$$

$$
\begin{gathered}
f(z)=U(s(z)), \quad g(z)=V(s(z)), \\
U, V \text { rational functions. }
\end{gathered}
$$

Suppose $R(u, v)$ is of degree $m_{1}$ in $u, n_{1}$ in $v$. Then for a given value of $v, u$ has in general $m_{1}$ possible values, i.e., there are $m_{1}$ values of $s$ for given $v$, i.e., $V$ is of degree $m_{1}$. Similarly $U$ is of degree $n_{1}$.

Since $f(z)$ and $g(z)$ are entire, $U$ and $V$ cannot have poles at any value taken on by $s(z)$. Since $s(z)$ omits at most one finite value, $U$ and $V$ can have poles at one finite value $s_{0}$ at most and then $s(z) \neq s_{0} \quad(z \in C)$. Without loss of generality we may suppose $s_{0}=0$ (otherwise replace $s$ by $s-s_{0}$ ). Combining all the information on $U$ and $V$ we find that we must have

By (10),

$$
\begin{array}{cc}
U(s)=\sum_{-v}^{n_{1}-v} a_{k} s^{k}, & V(s)=\sum_{-\mu}^{m_{1}-\mu} b_{k} s^{k}, \\
0 \leqq v \leqq n_{1} \leqq n, & 0 \leqq \mu \leqq m_{1} \leqq m .
\end{array}
$$

$$
P_{m} \circ U \circ s(z)=P_{n} \circ V \circ s(z) \quad(z \in C) .
$$

Since $s(z)$ takes on infinitely many distinct values, this implies

$$
P_{m} \circ U(s)=P_{n} \circ V(s) \quad(s \in C) .
$$


For large values of $s$, by (11) and $\left(12^{\prime}\right)$

Therefore

$$
P_{m} \circ U(s) \sim \text { const. } s^{\left(n_{1}-v\right) m}, \quad P_{n} \circ V(s) \sim \text { const. } s^{\left(m_{1}-\mu\right) n} .
$$

$$
\left(n_{1}-v\right) m=\left(m_{1}-\mu\right) n \text {. }
$$

Since $0 \leqq n_{1}-v \leqq n, 0 \leqq m_{1}-\mu \leqq m,(m, n)=1$, we must have

either $n_{1}-v=n, m_{1}-\mu=m ; n_{1}=n, v=0, m_{1}=m, \mu=0$

or $n_{1}=v, m_{1}=\mu$.

That is to say that either $U$ and $V$ are polynomials of degree $n$ and $m$ respectively or they are polynomials in $1 / s$. In this case $s(z) \neq 0 \quad(z \in C)$. Put $1 / s(z)=t(z)$. Arguing with the polynomials $U_{1}(t)=U(s), V_{1}(t)=V(s)$ we obtain again that $\left(12^{\prime}\right)$ implies $n_{1}=n, m_{1}=m$ and the proof of the Lemma is completed.

Lemma 1 reduces the study of the identity (10) to the investigation of polynomials $P_{m}, P_{n}, U_{n}, V_{m}$ satisfying

$$
P_{m} \circ U_{n}=P_{n} \circ V_{m} \quad(n, m)=1 .
$$

This relation was the subject of a beautiful and deep investigation by $\mathbf{J}$. F. Ritt in his paper [5]. The results of Ritt are summarized by

Lemma 2. The equation (13) can only hold under the following circumstances:

(A) There are first degree polynomials $\lambda, x, v, \mu$ such that

$$
\begin{gathered}
\lambda \circ P_{m} \circ \varkappa=T_{m}, \quad \lambda \circ P_{n} \circ v=T_{n}, \\
\varkappa^{-1} \circ U_{n} \circ \mu=T_{n}, \quad v^{-1} \circ V_{m} \circ \mu=T_{m}, \\
\lambda \circ P_{m} \circ U_{n} \circ \mu=\lambda \circ P_{n} \circ V_{m} \circ \mu=T_{n m},
\end{gathered}
$$

where the polynomials $T$ are defined by (4).

(B) Suppose $m>n$. There are first degree polynomials $\lambda, \varkappa, v, \mu$ and a polynomial $h(u)$ of degree $<m / n$ such that

$$
\begin{gathered}
\lambda \circ P_{m} \circ x(u)=u^{r} h^{n}(u) \quad(r+n \operatorname{deg} h=m), \\
\varkappa^{-1} \circ U_{n} \circ \mu(s)=s^{n}, \\
\lambda \circ P_{n} \circ v(u)=u^{n}, \\
v^{-1} \circ V_{m} \circ \mu(s)=s^{r} h(s)^{n}, \\
\lambda \circ P_{m} \circ U_{n} \circ \mu(s)=\lambda \circ P_{n} \circ V_{m} \circ \mu(s)=\left(s^{r} h\left(s^{n}\right)\right)^{n} .
\end{gathered}
$$

Lemma 3. Unless the polynomial $Q$ of degree $p$ is of the form

$$
Q(u)=A(u-\alpha)^{p}+B,
$$

there are only a finite number of pairs of first degree polynomials $v, \mu$ such that

$$
\nu \circ Q=Q \circ \mu \text {. }
$$


Proof. Let $v(t)=a t+b, \mu(t)=c t+d$. If

then

$$
v \circ Q=a Q(u)+b=Q \circ \mu=Q(c u+d),
$$

$$
a Q^{\prime}(u)=c Q^{\prime}(c u+d) .
$$

Therefore the set $S$ of zeros of $Q^{\prime}$ is invariant under the map $u \mapsto c u+d$. Since no translation leaves a finite set invariant, $c \neq 1$, unless $v(u)=\mu(u)=u$. If $c \neq 1$ we can write

$$
\mu(t)=c(t-\alpha)+\alpha, \quad \alpha=d /(1-c) .
$$

The invariance of $S$ now requires $|c|=1$, unless $S=\{\alpha\}$. (Consider a value of $t \in S$ for which $|t-\alpha|$ is maximal.)

If $S \neq\{\alpha\}$, then $c$ must be a root of unity, $c^{N}=1, c^{k} \neq 1 \quad(0<k<N)$. $S$ consists of corners of some regular $N$-gons with center $\alpha$ and possibly also $u=\alpha$. Hence

and, by integration,

$$
Q^{\prime}(u)=C(u-\alpha)^{s} \prod_{j=1}^{M}\left\{(u-\alpha)^{N}-b_{j}\right\}
$$

where $h$ is a polynomial.

$$
Q(u)=(u-\alpha)^{s+1} h\left[(u-\alpha)^{N}\right]+B
$$

$$
\begin{gathered}
Q \circ \mu=c^{s+1} Q(u)+B\left(1-c^{s+1}\right)=v \circ Q, \\
v(t)=c^{s+1} t+B\left(1-c^{s+1}\right), \quad\left(c^{N}=1\right) .
\end{gathered}
$$

There are only a finite number of possibilities for $\mu$ and $v$ in this case.

Finally, if $S=\{\alpha\}$, then

$$
Q^{\prime}(u)=C(u-\alpha)^{p-1}, \quad Q(u)=A(u-\alpha)^{p}+B .
$$

In this case any pair $\mu, v$ with

$$
\mu(t)=c(t-\alpha)+\alpha, \quad v(t)=c^{p} t+B\left(1-c^{p}\right) \quad(c \neq 1)
$$

is possible.

3. Proof of Theorem 2. Without loss of generality we may suppose that (1) holds for $k=n_{1}, q, n_{2}, n_{3}, \ldots$ where

$$
\left(n_{j}, q\right)=1 ; 1<n_{1}<q<n_{2}<n_{3} \ldots ; n_{j+1} \leqq n_{j} q .
$$

Using Lemma 1 with $m=n_{j}(j \geqq 2), n=q$ we see that there are polynomials $U$ (of degree $q$ ), $V$ (of degree $m$ ) and an entire function $s_{m}(z)$ such that

$$
P_{m} \circ U \circ s_{m}(z)=P_{q} \circ V \circ s_{m}(z)=F(z) \text {. }
$$

Now Lemma 2 shows that $P_{m}, U, P_{q}, V$ must be given either by the formulae (A) of Lemma 2 or by the formulae (B).

We show next that if (B) holds for a pair $m=n_{j}, n=q$, then (B) holds also for any other pair $\left(n_{k}, q\right)(k \geqq 1)$. 
Suppose we were in case (A) for $\left(n_{k}, q\right)$. Then we can find first degree polynomials $\varrho, \sigma$ such that

$$
\varrho \circ P_{q} \circ \sigma(u)=T_{q}(u) .
$$

On the other hand (B) for $m$ and $n=q<m$ shows that there are first degree polynomials $x, \lambda$ such that

Hence

$$
\lambda \circ P_{q} \circ \varkappa(u)=v^{q} \circ u .
$$

$$
\varrho \circ \lambda^{-1} \circ v^{q} \circ x^{-1} \circ \sigma(u)=T_{q}(u),
$$

or, writing out the first degree polynomials

$$
A(B u+C)^{q}+D=T_{q}(u) .
$$

But $T_{q}$ does not have any values of multiplicity $>2$. This leads to a contradiction, since $q \geqq 3$.

Theorem 2 will therefore be a consequence of the two statements:

(C) If there is an infinite sequence $M=\left\{m_{k}\right\}_{k=1}^{\infty}$ and a $q \geqq 3$ prime to all $m_{k}$ such that (16) and (A) of Lemma 2 hold, then $F(z)$ is of the form (3).

(D) If, for a sequence $m=n_{j}$, where the $n_{j}$ satisfy (15), (16) and (B) of Lemma 2 hold, then $F(z)$ is of the form (2).

Proof of (C). By (A) there are first degree polynomials $\lambda=\lambda_{m}, v=v_{m}$ such that

$$
\lambda \circ P_{q} \circ v=T_{q}
$$

If $\tilde{\lambda}$ and $\tilde{v}$ are the first degree polynomials corresponding to another value $\tilde{m} \in M$, then

$$
\lambda \circ \tilde{\lambda}^{-1} \circ T_{q} \circ \tilde{v}^{-1} \circ v=T_{q} .
$$

For $q \geqq 3 T_{q}(u)$ is not of the form $A(u-\alpha)^{p}+B$. By Lemma 3 there are only a finite number of possible values of the pair $\left(\lambda \circ \tilde{\lambda}^{-1}, \tilde{v}^{-1} \circ v\right)$. Keeping $m$ fixed and replacing $M$ by a subsequence, if necessary, we may assume that $\lambda$ does not depend on the choice of $m$. Formulae (16) and (A) now show that there is a first degree polynomial $\lambda$ such that

$$
\lambda \circ F(z)=T_{q m}\left(S_{m}(z)\right) \quad(m \in M),
$$

where $S_{m}(z)$ is an entire function $\left(S_{m}\right.$ is the composition of $s_{m}(z)$ in (16) with a first degree polynomial). Put

$$
S_{m}(z)=\cos \varphi(z)
$$

so that

$$
\lambda \circ F(z)=\cos q m \varphi(z) .
$$

The expression $\varphi(z)$ is not uniquely determined, but in a disk $U$ of the $z$-plane which contains no roots of $S_{m}(z)= \pm 1$ we can define $\varphi(z)$ as a holomorphic func- 
tion equal to a branch of $\operatorname{arc} \cos S_{m}(z)$. All possible values of $\varphi(z)$ in $U$ are obtained from one, $\varphi_{0}(z)$, say, by the formula

$$
\varphi(z)= \pm \varphi_{0}(z) \quad(\bmod 2 \pi) \quad(z \in U)
$$

Replacing $m$ by another member $\tilde{m}$ of the sequence $M$ we can similarly define $\psi(z)$ by

$$
\begin{gathered}
S_{\tilde{m}}(z)=\cos \psi(z), \\
\lambda \circ F(z)=\cos q \tilde{m} \psi(z) .
\end{gathered}
$$

Again $\psi$ is not uniquely defined, but in a disk in which $S_{\tilde{m}}(z) \neq \pm 1 \psi$ can be chosen as a holomorphic function. We may assume without loss of generality that this disk is identical with $U$.

Again all possible determination of $\psi$ can be derived from one of them, $\psi_{0}$, by the formula

$$
\psi(z)= \pm \psi_{0}(z) \quad(\bmod 2 \pi) \quad(z \in U)
$$

By (18) and (20),

$$
q \tilde{m} \psi_{0}(z) \equiv \pm q m \varphi_{0}(z) \quad(\bmod 2 \pi)
$$

Changing $\psi_{0}$ into $-\psi_{0}$, if necessary, we may suppose

$$
\begin{gathered}
q \tilde{m} \psi_{0}(z)=q m \varphi_{0}+2 h \pi, \\
\psi_{0}(z)=(m / \tilde{m}) \varphi_{0}(z)+2 h \pi / q \tilde{m} .
\end{gathered}
$$

Changing $\psi_{0}$ by adding a suitable multiple of $2 \pi$ we have

$$
\psi_{0}(z)=(m / \tilde{m}) \varphi_{0}(z)+c,
$$

where $c$ is a real number satisfying

$$
-\pi<c \leqq \pi
$$

Next we observe that the functions $\varphi_{0}$ and $\psi_{0}$ can be analytically continued along any path $C$ which avoids the roots of $S_{m}(z)= \pm 1, S_{\tilde{m}}(z)= \pm 1$. If $C$ is chosen as a closed path from a point in $U$ to $U$, then the results of the continuation $\varphi_{C}, \psi_{C}$ still satisfy (17), (19) and (22) with $\varphi_{0}$ and $\psi_{0}$ replaced by $\varphi_{C}$ and $\psi_{C}$. Let

Then

$$
\varphi_{C}(z)=\varepsilon \varphi_{0}(z)+2 l \pi \quad(\varepsilon= \pm 1) .
$$

$$
\psi_{C}(z)=(m / \tilde{m}) \varphi_{C}(z)+c=\varepsilon(m / \tilde{m}) \varphi_{0}(z)+2 m l \pi / \tilde{m}+c .
$$

Also

$$
\psi_{c}(z)=\eta \psi_{0}(z)+2 k \pi=\eta(m / \tilde{m}) \varphi_{0}(z)+\eta c+2 k \pi \quad(\eta= \pm 1)
$$


Note that $k$ depends on $\tilde{m}$ and $C, l$ on $m$ and $C$, and $c$ on $m$ and $\tilde{m}$ only. fore

Comparing (24) and (25) we see that $\varepsilon=\eta$, since $\varphi_{0}(z)$ is not constant. There-

$$
c+2 \operatorname{lm} \pi / \tilde{m}=\eta c+2 k \pi \text {. }
$$

If $\eta=1$ this reduces to

$$
\operatorname{lm} / \tilde{m}=k
$$

and for sufficiently large $\tilde{m}$ we must have $k=0$ and therefore also $l=0$. If $\eta=-1$, then

$$
2 k \pi=2 c+2 \operatorname{lm} \pi / \tilde{m} .
$$

For large $\tilde{m}(26)$ and (23) imply

$$
k=-1 \text { or } 0 \text { or } 1 \text {. }
$$

And since $c$ is independent of the path $C$, so $k$ must be independent of $C$, provided $\eta=-1$. We see that $\psi_{C}$ is only capable of assuming four values:

$$
\text { If } k=0, \quad \psi_{C}=\psi_{0} \quad(\eta=1) \quad \text { or } \quad \psi_{C}=-\psi_{0} \quad(\eta=-1) \text {. }
$$

(ii) If $k=1, \psi_{c}=2 \pi-\psi_{0}$.

$$
\text { If } k=-1, \quad \psi_{C}=-2 \pi-\psi_{0} \text {. }
$$

In the three cases respectively the functions

$$
\psi^{2},(\psi-\pi)^{2},(\psi+\pi)^{2}
$$

are single-valued functions of $z$, holomorphic at all points where $S_{\tilde{m}}(z) \neq \pm 1$. As $z$ approaches a root of this equation $\psi$, which is locally defined as a branch of $\operatorname{arc} \cos S_{\tilde{m}}(z)$, approaches a finite limit. The roots of $S_{\tilde{m}}(z)= \pm 1$ are therefore removable singularities of one of the functions (27). In case (i) we have

$$
\psi^{2}(z)=H(z) /(q \tilde{m})^{2}=\text { entire function }
$$

and $\lambda \circ F(z)=\cos \sqrt{H(z)}$ is of the form (3). In the other cases

$$
\begin{gathered}
\psi \pm \pi=\sqrt{H(z)} / q \tilde{m} \\
\lambda \circ F(z)=\cos (\mp q \tilde{m} \pi+\sqrt{H(z)})= \pm \cos \sqrt{H(z)}
\end{gathered}
$$

and again $F(z)$ is of the form (3).

Proof of (D). By (14) and (16) with $m=n_{k}, n=q$, there is a first degree polynomial $\lambda_{n}$ and an entire function $S_{k}(z)=A_{k} s_{n_{k}}(z)+B_{k}(A, B \in C)$ such that

$$
\lambda_{k} F(z)=\left[S_{k^{k}}^{r_{k}} h_{k}\left(S_{k}^{q}\right)\right]^{q} \quad(k=2,3, \ldots),
$$


where $h_{k}$ is a polynomial, $h_{k}(0) \neq 0$, and

$$
r_{k}+q \operatorname{deg} h_{k}=n_{k} \text {. }
$$

By replacing $S_{k}$ by $c S_{k}$, if necessary, we may assume

$$
h_{k}(0)=1 \text {. }
$$

Also, using (14) for the indices $n_{1}$ and $q$,

$$
\begin{gathered}
\lambda_{0} \circ F(z)=\left[S^{r} h\left(S^{n_{1}}\right)\right]^{n_{1}}, \\
r+n_{1} \operatorname{deg} h=q .
\end{gathered}
$$

If $\alpha$ is the root of $\lambda_{0}(t)=0, \beta$ the root of $\lambda_{k}(t)=0$; then every root of $F(z)=\alpha$, has multiplicity $\geqq n_{1}$, every root of $F(z)=\beta$ has multiplicity $\geqq q$, by (28) and (29). By Nevanlinna's 2nd fundamental theorem [1, p. 43], if $\alpha \neq \beta$

$$
T(r, F) \leqq \bar{N}(r, F)+\bar{N}\left(r, \frac{1}{F-\alpha}\right)+\bar{N}\left(r, \frac{1}{F-\beta}\right)+o(T(r, F)),
$$

as

$$
\begin{gathered}
\bar{N}(r, F)=0, \quad \bar{N}\left(r, \frac{1}{F-\alpha}\right) \leqq \frac{1}{n_{1}} N\left(r, \frac{1}{F-\alpha}\right) \leqq \frac{1}{n_{1}} T(r, F)(1+o(1)), \\
\bar{N}\left(r, \frac{1}{F-\beta}\right) \leqq \frac{1}{q} N\left(r, \frac{1}{F-\beta}\right) \leqq \frac{1}{q} T(r, F)(1+o(1)) .
\end{gathered}
$$

Therefore (30) leads to the contradiction

$$
T(r, F) \leqq\left(\frac{1}{q}+\frac{1}{n_{1}}+o(1)\right) T(r, F) .
$$

This contradiction can only be avoided, if $\alpha=\beta$, i.e., if $\lambda_{k}(t)=c \lambda_{0}(t)$. Replacing $S_{k}(z)$ by $b S_{k}(z)$ with a suitable $b$, we may suppose that

$$
\begin{gathered}
\lambda_{k}=\lambda_{0} \quad(k=2,3, \ldots,), \\
F_{0}(z)=\lambda_{0} \circ F(z)=\left(S_{k^{k}}^{r_{k}} h_{k}\left(S_{k}^{q}\right)\right)^{q}=\left(S^{r} h\left(S^{n_{1}}\right)\right)^{n_{1}} .
\end{gathered}
$$

Since $\left(n_{1}, q\right)=1$, (31) implies that $F_{0}(z)=0$ has only roots whose multiplicity is divisible by $n_{1} q$;

$$
F_{0}(z)=(G(z))^{n_{1} q},
$$

$G(z)$ entire. By (29) we can choose $G$ so that

Suppose

$$
G^{q}=S^{r} h\left(S^{n_{1}}\right)
$$

$$
h(t)=\prod_{\gamma}\left(t-\gamma^{n_{1}}\right)^{\mu(\gamma)} .
$$


Then $h\left(t^{n_{1}}\right)=\prod_{\gamma} \prod_{j=1}^{n_{1}}\left(t-\varrho^{j} \gamma\right)^{\mu(\gamma)}$, where $\varrho$ is a primitive $n_{1}$-th root of unity. If $z_{1}$ is a root of $S(z)=\varrho^{j} \gamma$ of multiplicity $v$, then $q \mid v \cdot \mu(\gamma)$. If $q \nmid \mu(\gamma)$, then $v \geqq 2$, i.e., the value $\varrho^{j} \gamma$ of $S$ is completely ramified. Also $r+n_{1} \operatorname{deg} h=q$. Therefore $0<r<q$ and each root of $S=0$ has multiplicity $\geqq 2$, by the preceding argument.

We have at least 3 ramified values of $S: 0, \gamma, \varrho \gamma$, if $\operatorname{deg} h>0$ and not all roots of $h$ have multiplicity divisible by $q$. Since an entire function has at most 2 ramified values we have either

$$
S^{r} h\left(S^{n_{1}}\right)=S^{q}
$$

or $q \mid \mu(\gamma)$ for all $\gamma$,

But

$$
S^{r} h\left(S^{n_{1}}\right)=S^{r_{1}}\left(k\left(s^{n}\right)\right)^{q} .
$$

$$
q=r+n_{1} \operatorname{deg} h_{1}=r+n_{1} q \operatorname{deg} k ;
$$

this is impossible, if $\operatorname{deg} k>0$, because $n_{1}>1$. Hence (32) holds and, by (29),

By (31) with $\mathrm{k}=2$

$$
F_{0}(z)=S^{n_{1} q} .
$$

$$
\left(S_{2}^{r_{2}} h_{2}\left(S_{2}^{q}\right)\right)^{q}=S^{n_{1} q}
$$

and we may suppose

$$
S_{2}^{r_{2}} h_{2}\left(S_{2}^{q}\right)=S_{1}^{n_{1}}, r_{2}+q \operatorname{deg} h_{2}=n_{2} .
$$

By repeating the reasoning above with $S_{2}$ in place of $S_{1}, S_{1}$ in place of $G$ and $n_{1}$ and $q$ interchanged we find

$$
\begin{gathered}
h_{2}\left(S_{2}^{q}\right)=\left(k_{2}\left(S_{2}^{q}\right)\right)^{n_{1}}, \\
r_{2}+n_{1} q \operatorname{deg} k_{2}=n_{2} .
\end{gathered}
$$

Since $\left(n_{2}, q\right)=1, \dot{r}_{2}>0$, and since $n_{2} \leqq n_{1} q$, we must have

Therefore

$$
S_{2}^{r_{2}} h_{2}\left(S_{2}^{q}\right)=S_{2}^{n_{2}} \text {. }
$$

$$
F_{0}(z)=\left(S_{3}^{r_{3}} h_{3}\left(S_{3}^{q}\right)\right)^{q}=S_{2}^{n_{2} q}
$$

which leads by the same reasoning to

$$
F_{0}(z)=S_{3}^{n_{3} q}=S_{4}^{n_{4} q} \ldots
$$

If $F_{0}$ has a root we arrive at a contradiction as soon as $n_{k} q$ is greater than the multiplicity of the root. Therefore

$$
F_{0}(z)=\lambda_{1} \circ F(z)=e^{H(z)},
$$

$H$ entire. $F(z)$ is of the form (2). 


\section{References}

[1] Hayman, W. K.: Meromorphic functions. - Clarendon Press, Oxford, 1964.

[2] OzAwA, M.: On a characterization of the exponential function and the cosine function by factorization. I, II, III. - Kodai Math. J. 1, 1978, 45-74, 313-315, and 2, 1979, 200-210, respectively.

[3] OzAwA, M.: On a characterization of the exponential function and the cosine function by factorization. IV. - Factorization theory of meromorphic functions and related topics, edited by C. C. Yang. Lecture Notes in Pure and Appl. Math. 78. Marcel Dekker, Inc., New York-Basel, 1982, 101-118.

[4] PiCARD, E.: Démonstration d'un théorème general sur les fonctions uniformes liées par une relation algébrique. - Acta Math. 11, 1887, 1-12.

[5] RitT, J. F.: Prime and composite polynomials. - Trans. Amer. Math. Soc. 23, 1922, 51-66.

Cornell University

Department of Mathematics

Ithaca, New York 14853

USA

Received 20 December 1983

\author{
East China Normal University \\ Shanghai \\ P. R. China
}

